\title{
Gravitational waves observation: brief comments
}

\author{
Observação de ondas gravitacionais: breves comentários \\ Mauro Cattani*1, José Maria Filardo Bassalo² \\ ${ }^{1}$ Instituto de Física, Universidade de São Paulo, São Paulo, SP, Brasil \\ ${ }^{2}$ Academia Paraense de Ciências, Belém, PA, Brasil
}

Recebido em 27 de Agosto, 2016. Aceito em 28 de Agosto, 2016

\begin{abstract}
In preceding papers we have shown the fundamental aspects of the General Relativity (GR), of the emission and detection of gravitational waves $(\mathrm{GW})$. With the same objective we analyze the two recent observations of the GW done by the Laser Interferometer Gravitational-Wave Observatory (LIGO) in the United States of America. These GW observations known as the GW150914 and GW151226 events are emitted by binary-star systems of black-holes (BBH). We present the basic principles of the laser interferometric technique that today is considered as the only one able to detect with certainty the GW. Using a simple relativistic approach we explain approximately the observed GW in the spiral stage.

Keywords: gravitational waves; laser interferometric technique; LIGO.
\end{abstract}

Em artigos precedentes analisamos os aspectos fundamentais da Relatividade Geral (RG), da emissão e detecção de ondas gravitacionais (OG). Com o mesmo objetivo analisaremos as duas recentes observações de OG feitas pelo Gravitational-Wave Observatory Laser Interferometer (LIGO) nos Estados Unidos. As observações dessas OG conhecidas como os eventos GW150914 e GW151226 são geradas por sistemasbinários de Buracos Negros (BBH). Apresentamos os princípios básicos da técnica de interferometria laser considerada hoje como a única capaz de detectar com certeza as OG. Com simples cálculos relativísticos explicamos aproximadamente as amplitudes das OG observadas na fase espiral.

Palavras-chave: ondas gravitacionais; técnica de interferometria laser; LIGO.

\section{Introduction}

It is important to begin this paper remembering that solving the Einstein's equations for a static distribution of mass $[1-4]$ we can obtain the unperturbed metric tensor $\mathrm{g}_{\mu \nu}^{(o)}$. When this mass distribution is slightly perturbed we write $\mathrm{g}_{\mu \nu}=\mathrm{g}_{\mu \nu}^{(o)}+\mathrm{h}_{\mu \nu}$ where $\mathrm{h}_{\mu \nu}$ represents a small correction of the initial gravitational field. In absence of matter $\mathrm{g}_{\mu \nu}^{(o)}$ is given by Minkowsky tensor $\mathrm{g}_{\mu \nu}^{(o)}=(1,1,1,-1)$. All relativistic accepted gravitation $[1-6]$ theories predict the existence of gravitational waves (GW) and that they are expected to have extremely small amplitudes with $\mathrm{h}_{\mu \nu} \sim 10^{-21}$. This can be seen in recent articles $[5,6]$, in which the fundamental equations of the Einstein gravitation theory are

*Endereço de correspondência: mcattani@if.usp.br constructed, experimental tests [5 - 7] are shown, and there are deductions of the basic equations predicting the emission of gravitational waves (GW) [8]. These equations have also been used to estimate the intensities, wave amplitudes (or strain) $\mathrm{h}_{\mu \nu}$ of the GW generated by some transmitter systems $[9,10]$ like, for instance,binary stars, neutron star pulsations, the precession of deformed neutron stars with oscillating quadrupoles, rotating bars and cataclysmic processes that give rise to supernovae. Many different detection techniques have been proposed to detect GW [3, 11,12]: laser interferometric techniques, resonant solids, fluctuations in the distance between Earth and Moon, crust oscillations of the Earth, normal modes of vibrations in solids in the form of rectangles, forks and rings, spinning rods and spinning tubes with fluids inside. In Section 2 we present the basic principles of the Laser 
Interferometry ${ }^{13}$ technique. Interferometers are the most sensible detecting systems: theoretically they are able to detect $\mathrm{GW}$ with amplitudes $\mathrm{h} \approx 3 \mathrm{X}$ $10^{-22}$, about 300 times smaller than estimates for any other detectors. In addition, another advantage of interferometers is that they can detect waves with any frequency. Only in 1990 it was arrived at the conclusion that GW could be detected with certainty only by interferometers [9, 10 - 12]. Detection of GW by another systems would be fortuitous. This occurred only after 20 years of intense researches with different techniques in USA, Japan, Italy, Australia, Holland,... Interferometric detectors were first suggested in the early 1960s and the 1970s [8 12]. In Section 3 we analyzed the two recent GW observations performed by the two interferometric detectors (LIGO) installed in the United States of America. These GW have been created in the merge of a binary black hole (BBH) system. As it will be seen in Section 2 the entire merge evolution of the BBH can be divided into three stages: "inspiral", "merger"(or "plunge") and "ringdown". To calculate exactly the GW emitted in the complete merge process it is necessary to solve the full equations of general relativity theory (GRT). This can be done only in numerical relativity simulations. Some simplified algebraic models can be used, for instance, in the "inspiral"stage. In Section 4, using a simple relativistic approach, we explain approximately the amplitude ("strain") of the GW signals detected by the LIGO in the "inspiral"stage. Of course, these estimations are non rigorous, only instructive.

\section{Laser interferometric technique}

The laser interferometric detector (LID) is basically a laser large Michelson [11 - 13] interferometer with perpendicular arms where there are three mirrors $\mathrm{M}$, M1 and M2 as shown in Figure 1. M1 and M2 mirrors are attached to suspended blocks that can swing freely like pendulums. With the aid of a highly potent monochromatic laser the interferometer measures the relative displacement of the mirrors which would be generated by GW.

Since 1990 several interferometric detectors were being built and designed: GEO600 (cooperation between Germany and England), LIGO (USA), VIRGO (collaboration between Italy and France), TAMA 300 (Japan), AIGO (Australia), LISA (spatial design, NASA, ESA) and LISC (LISA- in-

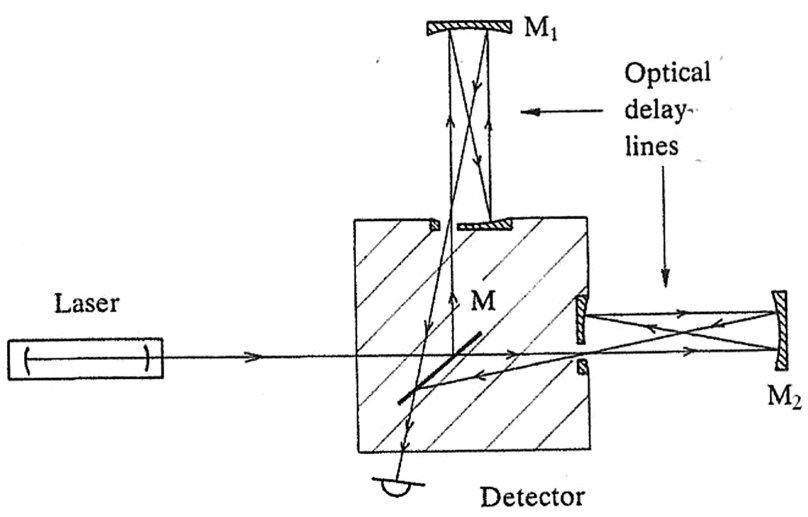

Figura 1: Scheme of a typical large Michelson interferometer to be used in the detection of gravitational waves.(Fig. 10.5 of Ref. 11)

ternational space project). As there is a huge amount of technical information involved in such major projects, we suggest to readers to search at Google using the words "Interferometric Detectors". The LIGO, VIRGO and GEO600 are very similar in concept. All projects have L-shaped facilities with multi-kilometer-long arms (4 km for LIGO, $3 \mathrm{~km}$ for VIRGO and $600 \mathrm{~m}$ for GEO600) with evacuated tubes that contain laser beams monitoring the positions of precision mirrors using interferometry. There is an intense collaboration between these international groups. According to Einstein's theory, the relative distance of the mirrors along the two arms changes very slightly when a GW passes by. The interferometers are set up in such a way that change in the lengths of the arms as small as $\sim$ $10^{-18} \mathrm{~m}$ (a thousandth the diameter of the atomic nucleus) can be detected.

Let us see the principle of operation of these detectors. Thus (see Fig.1) consider the GW incident on the $\mathrm{z}$-axis direction (perpendicular to the plane of the figure) and the polarizing axes $(+)$ along the $\mathrm{x}$-axis (passing through the laser, M and M2) and $\mathrm{y}$ (passing by M and M1). Following our article [6c], let us see how this GW changes the distance between two particles located along the $\mathrm{x}$ axis ( $\mathrm{y}$ $=\mathrm{z}=0)$ at a point $\mathrm{x}=-\mathrm{dx} / 2$, and the other at $\mathrm{x}=\mathrm{dx} / 2$. Supposing that the GW have a single polarization $(+)$ with an amplitude or strain $h$ the distance $\mathrm{d} \ell(\mathrm{x}, \mathrm{t})$ (calculated for delayed times $\mathrm{t}-\mathrm{xc}$ ) between the particles is given by,

$$
d \ell(x, t) \approx[1-(h / 2) \cos (\omega t-k x)] d x
$$


where $\mathrm{k}=\omega \mathrm{c}=2 \pi / \lambda$ and $\omega$ and $\lambda$, respectively, are the angular frequency and wavelength of the GW. Thus, if the points are separated by a finite distance with coordinates $\mathrm{x}= \pm \ell_{0} / 2$ from (1) we obtain the distance $\ell(\mathrm{t})$,

$$
\begin{aligned}
l(t) & =\int_{-l_{0} / 2}^{+l_{0} / 2}\left[1-\frac{h}{2} \cos (\omega t-k x) d x\right. \\
& =l_{0}-\frac{h}{2} \int_{-l_{0} / 2}^{+l_{0} / 2} \cos (\omega t-k x) d x
\end{aligned}
$$

where $\ell_{o}$ is the distance before the GW reach the particles. Thus, according to (2) the distance change $\Delta \ell(\mathrm{t})=\ell(\mathrm{t})-\ell_{o}$ generated by $\mathrm{G}$ is given by

$$
\begin{aligned}
\Delta \ell(t) & =(h / k) \cos (\omega t) \sin \left(k \ell_{o} / 2\right) \\
& =(\lambda h / 2 \pi) \cos (\omega t) \sin \left(\pi \ell_{o} / \lambda\right),
\end{aligned}
$$

showing that the amplitude $\Delta \ell$ of the displacement is given by

$$
\Delta \ell=(\lambda h / 2 \pi) \sin (\pi \ell o / \lambda)
$$

Under analogous conditions for the two points along the y axis, $\Delta \ell$ is also given by (4). Note that for $\ell_{0} \rightarrow 0$ the deformation amplitude is given by $\varepsilon=\Delta \ell / \ell \mathrm{o} \rightarrow \mathrm{h} / 2$, which is the shearing amplitude [5] along the $\mathrm{x}$ axis or $\mathrm{y}$ axis, as it is expected. The distance changes $\Delta \ell$ of these arms have opposite phases: while one arm shortens the other lengthens and vice versa $[6 \mathrm{c}]$.

According to (4) the maximum $\Delta \ell$ is obtained when $\ell_{o}=\lambda / 2$, that is, $(\Delta \ell)_{\max }=\lambda \mathrm{h} / 2 \pi$. This implies that a minimum amplitude $\mathrm{h}_{\min }$ of one GW which would be able to create $\Delta \ell$ is given by $\mathrm{h}_{\text {min }} \approx \Delta \ell /(\lambda / 2)$. Distance changes $\Delta \ell(\mathrm{t})$ create fluctuations in interference fringes. The laser light is divided into two parts by the mirror $\mathrm{M}$ which propagate along the interferometer arms. The light beams are reflected by M1 and M2, return, coherently recombine again in $\mathrm{M}$, and are detected by the photosensitive device. In (4) the distance $\ell_{o}$ would be the undisturbed "optical path length". From (4) we see that the maximum $\Delta \ell$ value is obtained when $\ell_{o}=\lambda / 2$, or $(\Delta \ell)_{\max }=\ell_{o} \mathrm{~h} / \pi$. For GW with $\mathrm{f}=1$ $\mathrm{kHz},(\Delta \ell)_{\max }$ is obtained when $\ell_{o}=150 \mathrm{~km}$. Multiple passes are made in each arm to increase the optical path thereby obtaining an "effective optical length "L. A simplified scheme is shown in Fig.1 with the beam back and forth between the mirrors by successive reflections. This process is known as "delay line"(optical delay line). Assuming $\mathrm{h}=10^{-21}$ and $\mathrm{f}=1 \mathrm{kHz}, \lambda=\mathrm{c} / \mathrm{f}=300 \mathrm{~km}$, we found that $(\Delta \ell)_{\max }=\mathrm{h} \lambda / 2 \pi=5 \times 10^{-15} \mathrm{~cm}$, i.e. 100 times less than the proton radius .

Due to the extremely small intensities of GW $[5,6]$, the interferometer must be optically perfect and extremely well isolated from the rest of the world (seismic isolation, cosmic rays, electromagnetic fields, etc.). Lasers must be very powerful, extremely monochromatic and stable, the mirrors must have high reflectivity, the light path must be done over extreme high vacuum tubes, etc.

The measurement accuracy is mainly limited by fluctuations in the interference fringes, the number of detected photons as they mimic the effect of changes in the optical path. Suppose at a point $\mathrm{P}$ (in the mirror $\mathrm{M}$ ) the intensity of the light from one arm is $\mathrm{A}$ and the intensity from the other arm is exp (i2 $\pi \Delta \mathrm{L} / \lambda_{\text {em }}$ ), where $\Delta \mathrm{L}=\mathrm{L}_{1}-\mathrm{L}_{2}$ is difference of the effective paths of the two light beams along the arms 1 and 2, respectively, and $\lambda_{e m}$ is the wavelength of the laser light. Thus, the light intensity at the detector is given by

$$
\begin{aligned}
& \left|A+A \exp \left(i 2 \pi \Delta L / \lambda_{e m}\right)\right|^{2} \\
& =4 A^{2} \cos ^{2}\left(\pi \Delta L / \lambda_{e m}\right) .
\end{aligned}
$$

The distribution of the number $\mathrm{N}$ of photons in the interference fringes is then given by [13]

$$
N=N^{\max } \cos ^{2}\left(\pi \Delta L / \lambda_{e m}\right) .
$$

Due to the incident GW the distances originally undisturbed $L_{1}$ and $L_{2}$ are changed by $\mathrm{dL}_{1}$ and $\mathrm{dL}_{2}$, respectively, resulting in a total variation $\mathrm{d}(\Delta \mathrm{L})=$ $\mathrm{dL}_{1}-\mathrm{dL}_{2}$. The effect of $\mathrm{d}(\Delta \mathrm{L})$ on $\mathrm{N}$ is given by

$$
d N=N_{\max }\left(\pi / \lambda_{\text {em }}\right) \sin \left(2 \pi \Delta L / \lambda_{\text {em }}\right) d(\Delta L) .
$$

As the statistical error of $\mathrm{N}$ is $\mathrm{N}^{1 / 2}$, the accuracy in the $\mathrm{d}(\Delta \mathrm{L})$ measurement can be estimated replacing $\mathrm{dN}$ by $\mathrm{N}^{1 / 2}$ into (7),

$$
d(\Delta L)=\lambda_{e m} N^{1 / 2} /\left[\pi N_{\max } \sin \left(2 \pi \Delta L / \lambda_{e m}\right)\right],
$$

where we see that $d(\Delta \mathrm{L})$ is minimum when sin $\left(2 \pi \Delta \mathrm{L} / \lambda_{e m}\right)=1$. With this condition (6) becomes 
written as $\mathrm{N}=\mathrm{N}_{\max } / 2=\mathrm{N}_{o}$, where $\mathrm{N}_{o}$ is the mean intensity. Under these conditions, (8) gives

$$
d(\Delta L)=\lambda_{e m} /\left(2 \pi N_{o}^{1 / 2}\right) .
$$

The coefficient $\mathrm{N}_{o}$ depends on the laser power $\mathrm{P}$, the time interval $\Delta \tau$ of the duration of the measurement, and the detection efficiency $\varepsilon$ of the photons according to equation $[7,9]$,

$$
N_{o}=\Delta \tau \varepsilon P /\left(\hbar \omega_{e m}\right),
$$

where $\omega_{e m}$ is the angular frequency of the laser light. Substituting (10) into (9) we have

$$
\begin{aligned}
& d(\Delta L)=\left(\lambda_{e m} / 2 \pi\right)\left(\hbar \omega_{e m} / \Delta \tau \varepsilon P\right)^{1 / 2} \\
& =\left[c^{2} \hbar / \omega_{e m} \Delta \tau \varepsilon P\right]^{1 / 2}
\end{aligned}
$$

Using (4) we have seen that the maximum amount of displacement due to the GW is achieved when the path length was equal to $\lambda / 2$. Under these conditions the photons remain a time $\lambda / 2 \mathrm{c}$ within the interferometer arm. This means that the measurement time $\Delta \tau$ must be equal to or greater than the travel time $\lambda / 2 \mathrm{c}$. So, putting $\Delta \tau \approx \lambda / 2 \mathrm{c}$ into (11) we get

$$
d(\Delta L)=\left(c \hbar \lambda_{e m} f / \pi \varepsilon P\right)^{1 / 2} .
$$

According to (4) we conclude that the minimum strain $\mathrm{h}_{\text {min }}$ of GW that generates a change $\mathrm{d}(\Delta \mathrm{L})$ in an effective length must obey the relationship $\mathrm{h}_{\text {min }}$ $=\mathrm{d}(\Delta \mathrm{L}) / \mathrm{L}=\mathrm{d}(\Delta \mathrm{L}) /(\lambda / 2)$ from which we obtain, using (12),

$$
h_{\text {min }}=2\left(\hbar \lambda_{e m} f^{3} / c \pi \varepsilon P\right)^{1 / 2} .
$$

Using (13) and $\mathrm{P}=100 \mathrm{~W}, \lambda_{\text {em }}=500 \mathrm{~nm}, \mathrm{f}=$ $1 \mathrm{kHz}$ and $\varepsilon=0.3$, we verify that $\mathbf{h}_{\min } \approx 310^{-22}$.

As in (13) we supposed that $\mathrm{f}=1 \mathrm{kHz}$ and the optical path $\mathrm{L}=\lambda / 2=\mathrm{c} / 2 \mathrm{f}=150 \mathrm{~km}$ the light ray must make a number $\mathrm{n}=150 / \mathrm{L}_{b}$ of passages where $\mathrm{L}_{b}$ is the length of the interferometer arm. In the arm the travel time of the photon $\Delta \tau$ is equal to $\Delta \tau$ $=\lambda / 2 \mathrm{c}=5 \mathrm{~ms}$. In VIRGO, for instance, as $\mathrm{L}_{b}=3$ $\mathrm{km}$ we verify that $\mathrm{n}=50$. In these conditions we see that the optical path variation $\mathrm{d}(\Delta \mathrm{L})=\mathrm{h}_{\text {min }} \mathrm{L}=$ $4.510^{-15} \mathrm{~cm}$ and the fluctuation $\mathrm{dN}$ in the number of photons is $\mathrm{dN} \approx \mathrm{N}_{o}^{1 / 2}$. According to (9), it is given by $\mathrm{dN}=\mathrm{N}_{o}^{1 / 2}=\lambda_{e m} /[2 \pi \mathrm{d}(\Delta \mathrm{L})] \sim 10^{10}$.

Note that the interferometers can in principle detect GW with any frequency. Eq.(13) is a simple estimation of the minimum strain $\mathrm{h}_{\text {min }}$ taking into account only a few factors such: $\lambda_{e m}$, laser power $\mathrm{P}, \varepsilon$ detection efficiency of the light signal, etc ... However, there are many other factors that limit sensitivity of interferometers and that depend on frequency $f$ of the GW. Very accurate estimates and simulations have been made to estimate the $\mathrm{h}_{\text {min }}$ by the observatories GEO 600, LIGO, VIRGO, TAMA 300, AIGO, LISA and LISC taking into account numerous effects. These are seismic, thermal and thermo-elastic in the pendulums that hold the mirrors, radiation pressure, acoustic, electromagnetic, cosmic rays, distortion of the surfaces of the mirrors due to laser coating of mirrors, "shot noise", etc. We believe instructive, from a didactical point of view, to display in one figure how reliable effects contribute to obtain the sensitivity curve sensitivity curve $\mathrm{h}_{\min }(\mathrm{f}) / \operatorname{sqrt}(\mathrm{Hz})$ as function of $\mathrm{f}[\mathrm{Hz}]$. This is the case of the VIRGO interferometer $[10,11]$ that was projected $[14,15]$ in 1990 and today is installed at Cascina (Pisa-Italy); each arm of the interferometer has $3 \mathrm{~km}$.

As it will be shown in Section 3, the current Advanced LIGO and VIRGO Interferometers are able to detect signals with $\mathrm{h}_{\text {min }} \approx 3 \times 10^{-22}$ which is theoretically 300 times smaller than the estimates for any other GW detectors [6 - 12].

\section{Observations of Gravitational Waves by LIGO}

As we have mentioned above, interferometric detectors were first suggested in the early 1960s and the 1970s. Long-baseline broadband laser interferometers began to be proposed in $1989-2000$. Combinations of these detectors made joint observations from 2002 through 2011, setting upper limits on a variety of gravitational-wave while LIGO and VIRGO became the first significantly more sensitive network of advanced detectors to begin observations $[16,17]$.

In Figure 2 it is shown a simplified diagram of the Advanced LIGO detector (see details in reference 12), a modified Michelson interferometer: each arm is formed by two mirrors connected to test masses, separated by $\mathrm{L}_{x}$ and $\mathrm{L}_{y}=\mathrm{L}=4 \mathrm{~km}$ (see reference 


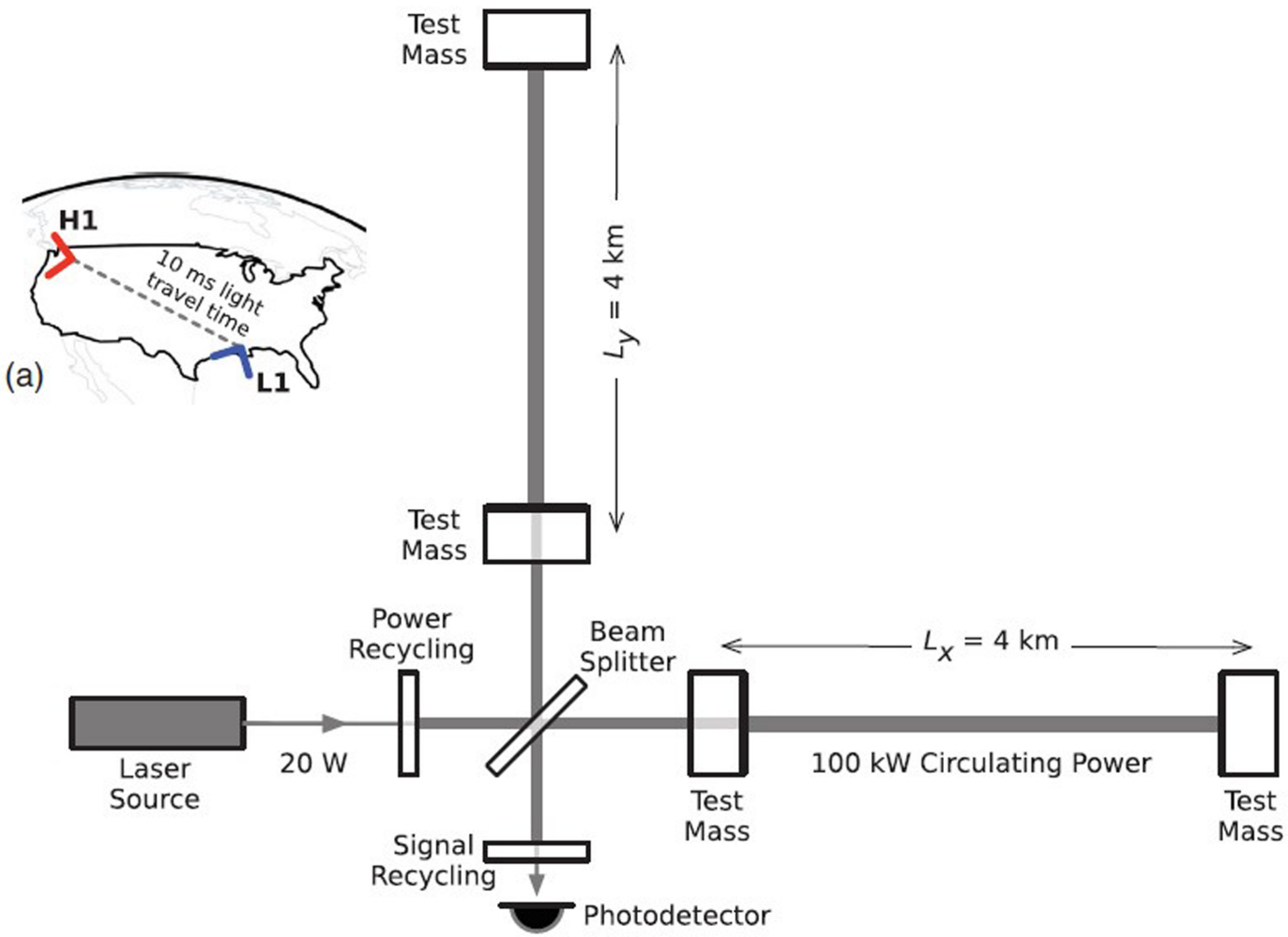

Figura 2: Simplified diagram of the Advanced LIGO detector not in scale with the arms along the axis $x$ and $y$ with lengths $\mathrm{L}_{x}=\mathrm{L}_{y}=\mathrm{L}=4 \mathrm{~km}$. Insert (a): locations of the LIGOs at Hanford (H1) and Livingstone (L1) distant $\sim 3,000 \mathrm{~km}$ with arms in different orientations. (Publicly available image at https://www.ligo.caltech.edu/)

$[16])$. In this figure is also shown the two identical LIGO detectors that have been constructed in USA : one at Hanford (H1) and another at Livingstone (L1), distant by $10 \mathrm{~ms}$ light $\sim 3000 \mathrm{~km}$ with arms in different orientations.

A passing gravitational wave (Figure 3) effectively alters the arm lengths such that the measured

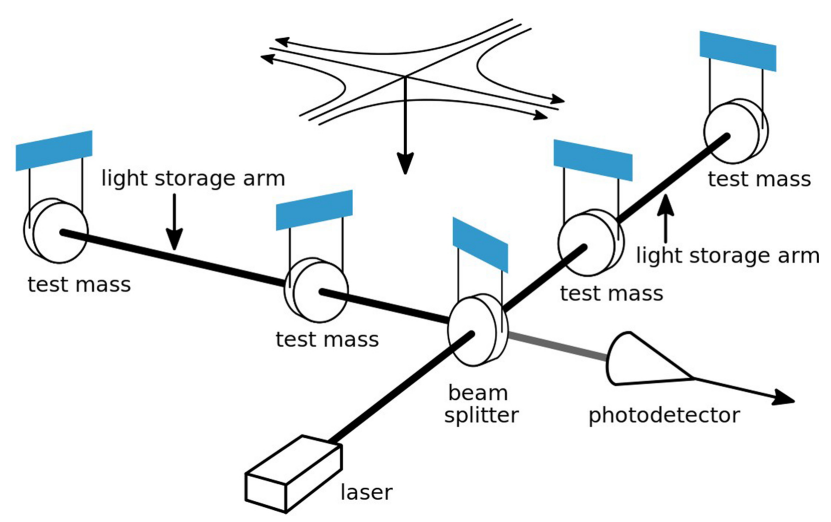

Figura 3: Schematic incidence of the plane GW on the Ligo detector. Note the tensorial nature of the incident GW given by $h_{\alpha \beta}(x, t)[4,8]$. (Publicly available image at https://www.ligo.caltech.edu/) difference is $\Delta \mathrm{L}(\mathrm{t})=\delta \mathrm{L}_{x}-\delta \mathrm{L}_{y}=\mathrm{h}(\mathrm{t}) \mathrm{L}$, where $\mathrm{h}(\mathrm{t})$ is the gravitational-wave strain.

Figure 4 shows for the (Advanced) LIGO the strain sensitivity $\mathrm{h}_{\min } / \sqrt{ } \mathrm{Hz}$ as a function of the gravitational frequency $\mathrm{f}$ in $\mathrm{Hz}$. LIGO detector is able to detect signals with $\mathrm{h}_{\min } \sim 7 \mathrm{X} 10^{-23}$.

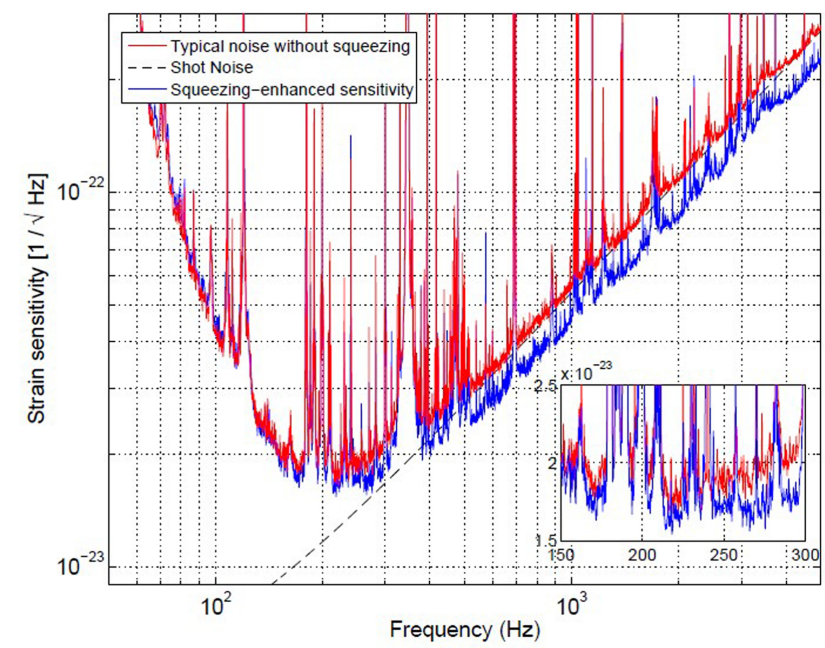

Figura 4: The strain sensitivity $\mathrm{h}_{\min } / \sqrt{ } \mathrm{Hz}$ for LIGO as a function of the gravitational frequency $\mathrm{f}$ in $\mathrm{Hz}$. (Publicly available image at https://www.ligo.caltech.edu/) 
The current strain sensitivity curves $\mathrm{h}_{\min } / \sqrt{ } \mathrm{Hz}$ for VIRGO and LIGO are very similar [18].

\subsection{Binary Black Hole (BBH)}

For many years, proving the existence of BBHs was quite difficult [19] because of nature of the BHs themselves, and the limited means of detection available. However, in one event that a pair of BHs were to merge, an immense amount of energy should be given off as GW, with distinct waveforms that can be calculated using the GR. Therefore, during the late 20th and early 21st centuries, BBHs became of great interest scientifically as a potential source of such waves, and a means by which gravitational waves could be proven to exist. BBH mergers would be one of the strongest known sources of gravitational waves in the Universe, and thus offer a good chance of directly detecting such waves [19]. These sources have been finally observed in the events known as GW150914 [16] and GW151226 [20].

The entire merge evolution of the $\mathrm{BBH}$ can be divided into three stages: "inspiral", "merger"(or "plunge") and "ringdown". The "inspiral" is the first stage of the BBH life which resembles a gradually shrinking orbit and takes a longer time, as the emitted GWs are very weak when the BHs are distant from each other. This phase contributes only with a small fraction of the GW energy emitted in the entire process. As the BH orbit shrinks, the speed increases, and the GW emission increases. When the BHs are close the GW, they cause the orbit to shrink rapidly. In the final fraction of a second the $\mathrm{BH}$ can reach extremely high velocity. This is followed by a plunging orbit and the $\mathrm{BH}$ will "merge" once they are close enough. At this time the GW amplitude reaches its peak (it is only clearly observed in the GW151226 event [20]). Once merged, the single hole settles down to a stable form, via a stage called "ringdown", where any distortion in the shape is dissipated as more gravitational waves. To calculate exactly the GW emitted in the complete merge process it is necessary to solve the full equations of general relativity. This can be done only in numerical relativity simulations $[16,17,19,20]$. Some simplified algebraic models can be used, for instance, in the "inspiral"stage. In Section 4 using the general relativity theory we estimate approximately the amplitude ("strain") of the GW signals detected by the LIGO in the "inspiral"stage.

\subsection{Event GW150914 observation}

The existence of stellar-mass BBHs (and gravitational waves themselves) was finally confirmed on September 14, 2015 at 09:50:45 UTC when the two LIGO detectors H1 and L1 have detected simultaneously a wave transient gravitational-wave signal. The signal sweeps upwards in frequency from 35 to $250 \mathrm{~Hz}$ with a peak gravitational-wave strain $\mathrm{h}=1.0 \times 10^{-21}$. This transient signal measured in H1 and L1 is seen in Figure 5 [12]. The signals $\mathrm{h}(\mathrm{t})$ can be divided into three phases : "inspiral", "merger"(or "plunge") and "ringdown", that will be clearly shown in Figure 7. The merging BBH with a pair of $\mathrm{BH}$ with masses $\sim 30 \mathrm{M}_{\Theta}$ was $\sim 1.3$ billion light years away and in its final moments of spiraling inward and merging released around $3 \mathrm{M}_{\Theta}$ as gravitational energy, peaking at a rate of $3.610^{49} \mathrm{~W}$ - more than the combined power of all light energy radiated by all stars on the observable universe put together [19, 20].

In Figure 6 we compare the strain $\mathrm{h}(\mathrm{t})$ measured by H1 and L1. We verify that they are very similar, in good agreement within the experimental errors.

Observing the evolution of the gravitational-wave signal $\mathrm{h}(\mathrm{t})$ one could deduce $[16,8]$ that the most plausible explanation for this is that a binary system formed by black holes $(\mathrm{BH})$ with masses $\mathrm{m}_{1}$ and $\mathrm{m}_{2}$ ( Figs. 7) initially moving in a circular orbit begin to move in spiral around the center of mass and finally merging. With this model the predicted strain $\mathrm{h}(\mathrm{t})$ of the "gravitational luminosity"was calculated by the LIGO collaborators and is shown in Fig. 7 compared with the observed strain that has a peak $|\mathrm{h}| \sim 10^{-21}$. Figure 7 clearly shows that the signals can be divided into three phases : "inspiral", "merger"and "ringdown". In the initial inspiral phase the BH move freely, in the merger phase they begin to "plunge"and, finally, the "ringdown"is the post-fusion phase.

\subsection{Event GW151226 observation}

On December 26, 2015 at 03:38:53 UTC the twin LIGO detectors H1 and L1 have detected simultaneously [20] a second wave transient gravitational-wave signal GW151226 shown in Figure 8. The signal persisted in the LIGO frequency band for approximately $1 \mathrm{~s}$, from $\mathrm{t}=-1.0$ up $\mathrm{t}=0$, increasing in frequency and amplitude over about 55 cycles from 35 to $450 \mathrm{~Hz}$, and reached a peak gravitational strain 


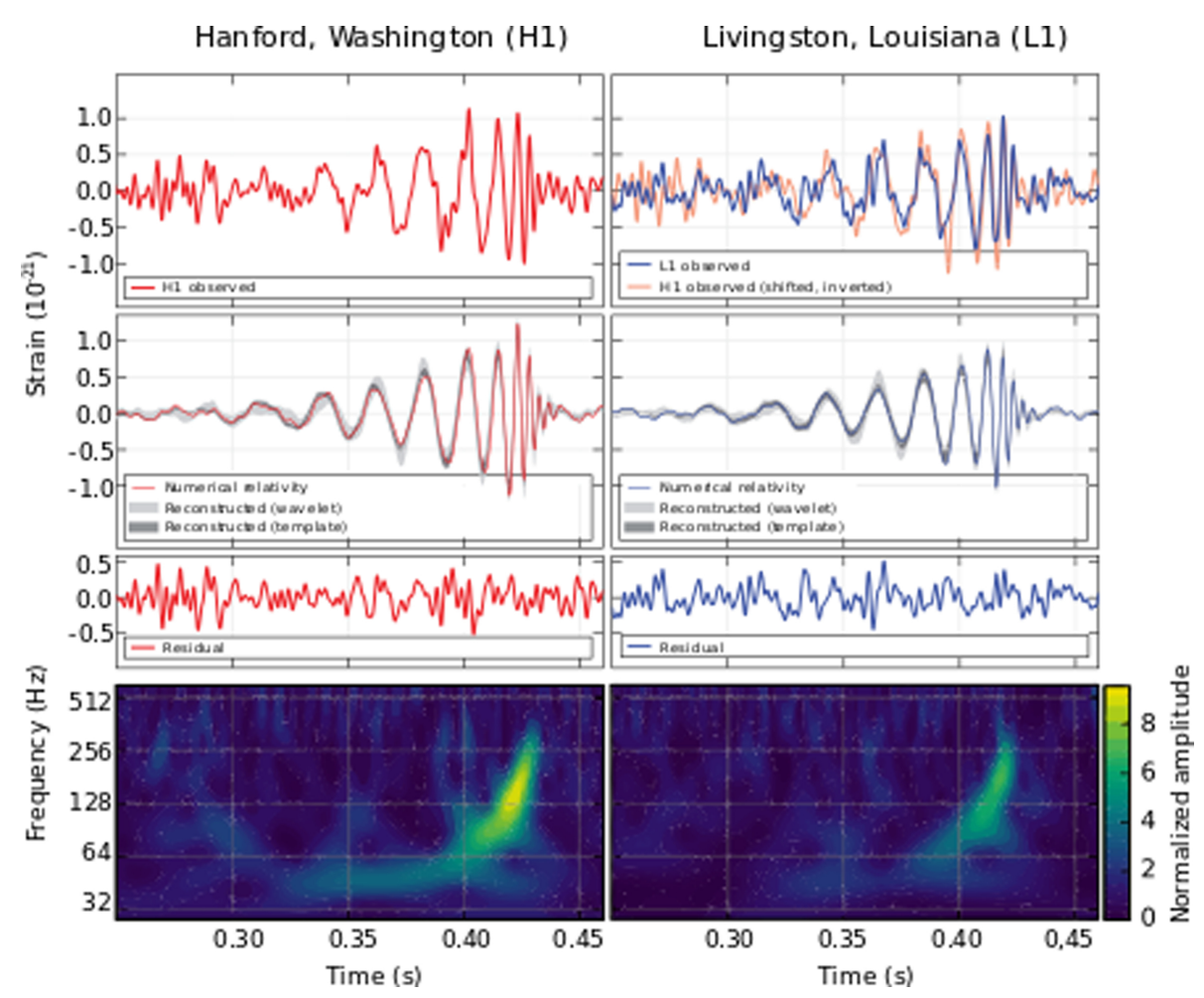

Figura 5: The gravitational-wave event detected simultaneously by the two LIGO interferometers H1 and L1 on September 14, 2015 at 09:50:45 UTC. Detailed description of this figure is given in reference 20. It is fundamental to note that the signals detected in $\mathrm{H} 1$ and $\mathrm{L} 1$ are, within the experimental errors, the same. Note that the frequencies $(\mathrm{Hz})$ shown in Fig.5 are the frequencies $\omega_{g}$ of the detected GW. (Publicly availble image at https://www.ligo.caltech.edu/)

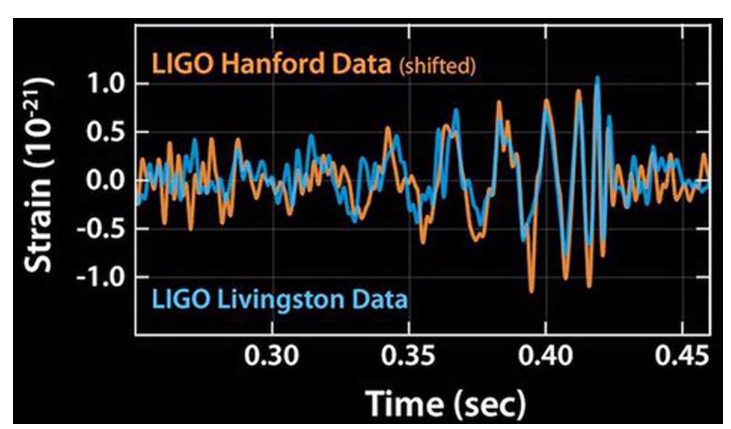

Figura 6: Comparison between the gravitational-wave signals $\mathrm{h}(\mathrm{t})$ measured by $\mathrm{H} 1$ and $\mathrm{L} 1 .^{16}$ (Publicly available image at https://www.ligo.caltech.edu/)

$|\mathrm{h}| \sim 3.4 \mathrm{X} 10^{-22}$. In this interval, that is, from $\mathrm{t}$ $=1.0 \mathrm{~s}$ up to $\mathrm{t} \approx 0 \mathrm{~s}$ the $\mathrm{BBH}$ is practically in the "inspiral"stage. At $\mathrm{t} \approx 0 \mathrm{~s}$ the BHs "merge"and the GW amplitude reaches its peak as can be seen in the third row of the Fig.8 [Signal-to-noise (SNR) time series]. For $t>0$, we have the "ringdown"stage.

Comparing Figs.5 -6 and 8 we see that they are very similar. That is, observing the evolution of the gravitational-wave signal $\mathrm{h}(\mathrm{t})$ one could also deduce that the most plausible explanation is that a $\mathrm{BBH}$ with masses $\mathrm{m}_{1} \approx 14.2 \mathrm{M}_{\Theta}$ and $\mathrm{m}_{1} \approx 7.5 \mathrm{M}_{\Theta}$ (see Fig.7) initially moving in a circular orbit begin to move in spiral around the center of mass and finally merging. With this model the predicted strain $\mathrm{h}(\mathrm{t})$ of the "gravitational luminosity"was calculated by the LIGO collaborators and is shown in Fig. 9. According to these estimations for three different times in the "inspiral"stage (seen in the first row of Fig. 9) we have $\mathrm{h} \sim 0.1710^{-21}, \mathrm{~h} \sim 0.1810^{-21}$ and finally, close to the merging point $\mathrm{t} \sim 0 \mathrm{~s}, \mathrm{~h} \sim 0.34$ $10^{-21}$.

From Figs.4-7 and Figs. 8,9 we observe a good agreement within the experimental errors between theory and measurements. Comparing orbital with observed GW frequencies one can see [16, 20] that the emitting objects of the binary systems can only be very compact $\mathrm{BH}$. These results confirm that GW have finally been detected and that they are generated by merging BBH system. In next Section using the GTR we estimate approximately the GW strain $\mathrm{h}(\mathrm{t})$ in the "inspiral"stage of a BBH system. 


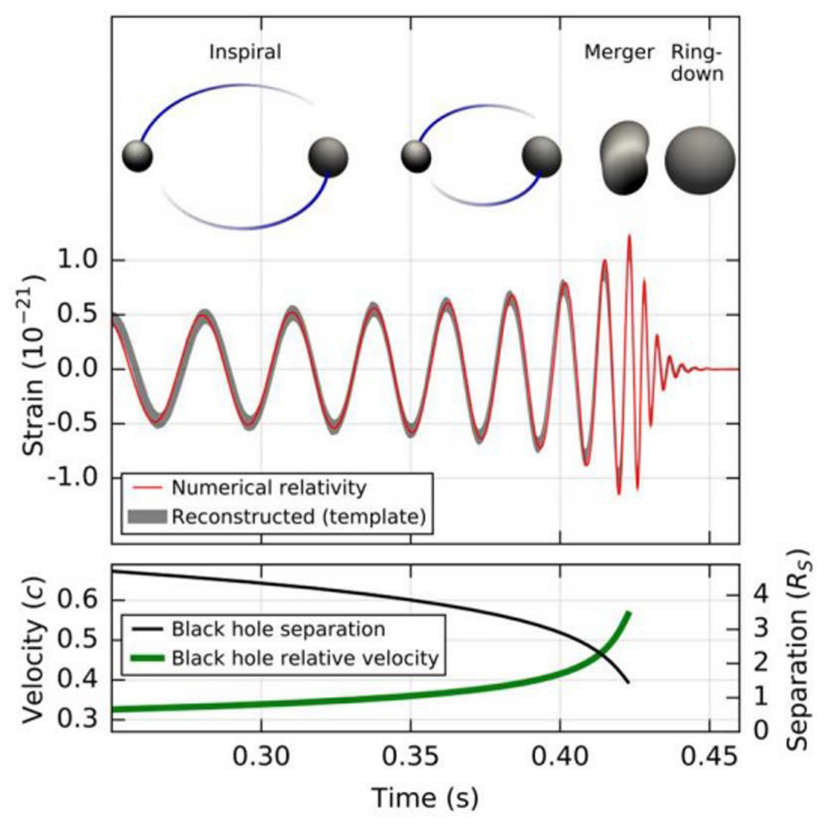

Figura 7: In order to explain the gravitational-wave strain $\mathrm{h}(\mathrm{t})$, we assumed that it was due to a binary system of two $\mathrm{BH}$ with masses $m_{1}$ and $m_{2}$ initially moving in a circular orbit that begin to move in spiral around the center of mass and finally merging [16]. (Publicly available image at https://www.ligo.caltech.edu/)

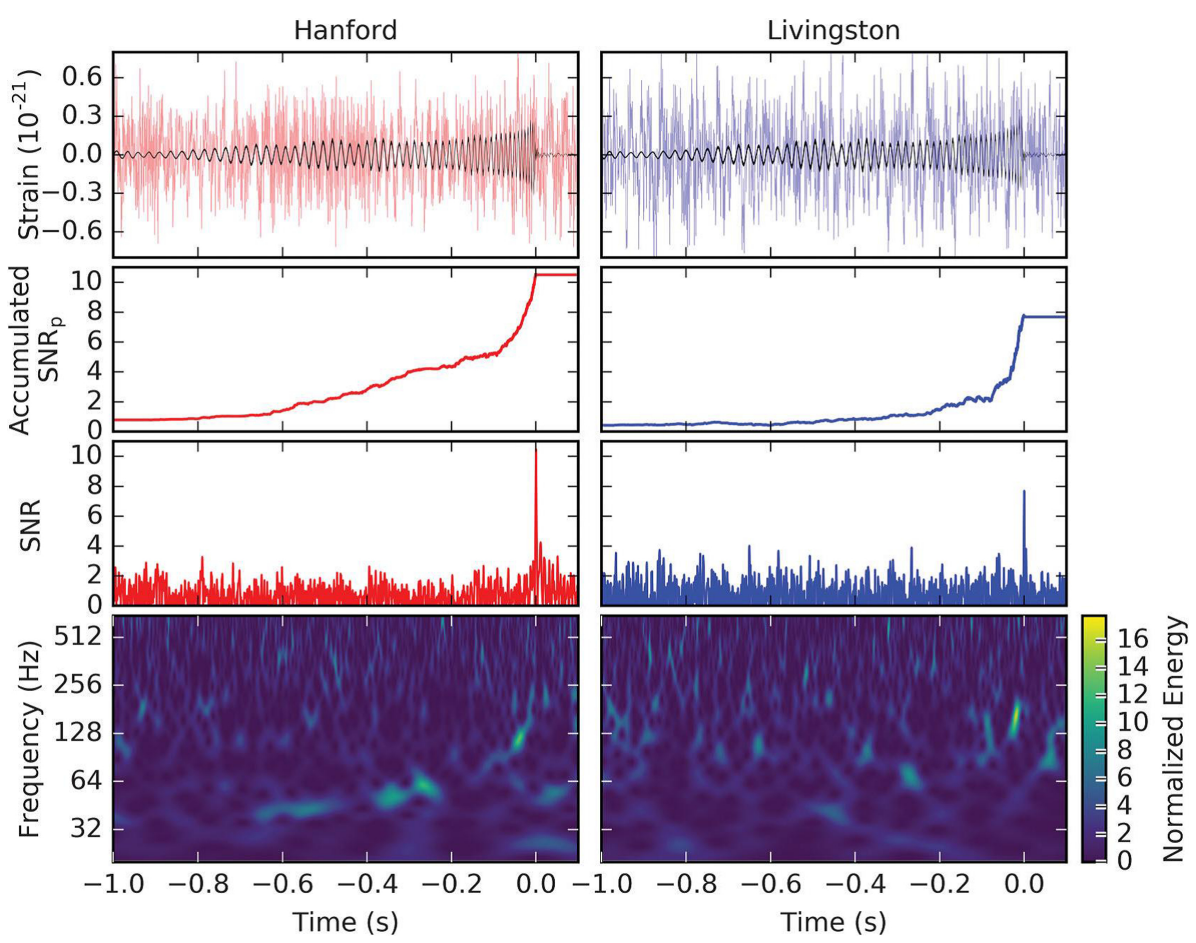

Figura 8: GW151226 observed by the LIGO Hanford (left column) and Livingston (right column) detectors, where times are relative to December 26, 2015 at 03:38:53.648 UTC. It is fundamental to note that the signals detected in H1 and L1 are, within the experimental errors, the same [20]. (Publicly available image at https://www.ligo.caltech.edu/)

\section{Strain estimation for a binary-star system}

We remark that our calculations are not rigorous; they have only a didactical objective. As we have said before, to describe exactly the detected signals of the GW in the complete merge BBH process of the BBH ("inspiral", "plunge"and "ringdown") it is necessary to use a completely relativistic model that can be treated only with numerical simulati- 


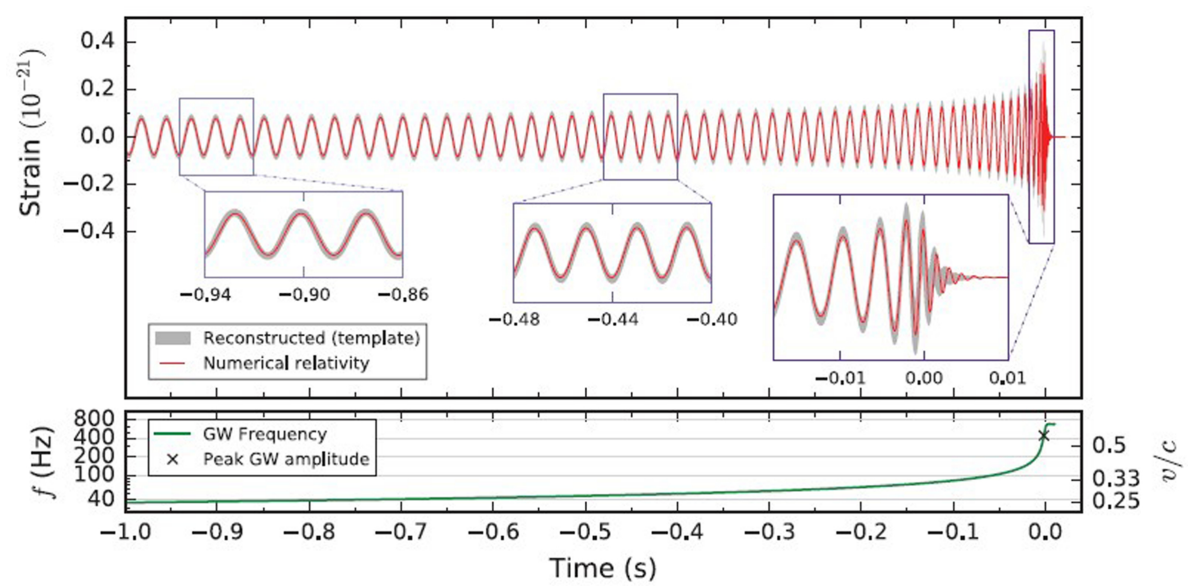

Figura 9: Estimated gravitational-wave strain from GW151226 projected onto the LIGO Livingston detector with times relative to December 26, 2015 at 03:38:53.648 UTC. To explain the gravitational-wave strain $\mathrm{h}(\mathrm{t})$, we assumed that is was due a binary system (two black holes) with masses $m_{1}$ and $m_{2}$ initially moving in a circular orbit that begin to move in spiral around the center of mass and finally merging. ${ }^{20}$ (Publicly available image at https://www.ligo.caltech.edu/)

ons $[16.17,19,20]$. Our approach describes only approximately the "inspiral"phase of the observed $\mathrm{BBH}$.

Let us begin calculating the GW strain $\mathrm{h}(\mathrm{t})$ emitted by a generic binary-star system. We take $\mathrm{R}$ as the distance between the detector and the GW source that has a dimension D. According to preceding papers $[4,8]$, the emitted GW in the radiation zone $(\mathrm{R} \gg \mathrm{D})$ is represented by the second-rank tensor

$$
h_{\alpha \beta}(t, x)=\frac{2 G}{c^{2} R} \frac{\partial^{2} Q_{\alpha \beta}}{\partial t^{2}}=\frac{2 G}{c^{2} R} \ddot{Q}_{\alpha \beta}
$$

where $\mathrm{R}$ is the radius and $\mathrm{Q}_{\alpha \beta}(\mathrm{t})$ is the tensor quadrupole moment of the source given by

$$
Q_{\alpha \beta}(t)=\int \rho_{o}\left(3 x^{\alpha} x^{\beta}-\delta_{\alpha \beta} r^{2}\right) d V
$$

For a binary system (Figure 10) composed by stars with masses $m_{1}$ and $m_{2}$ separated by a distance $r$ one can show [9] that

$$
\begin{aligned}
& Q_{x x}=3 \mu r^{2}[1+\cos (2 \omega t)] / 2 \text { and } \\
& Q_{y y}=3 \mu r^{2}[1-\cos (2 \omega t)] / 2
\end{aligned}
$$

where $\mu=\mathrm{m}_{1} \mathrm{~m}_{2} /\left(\mathrm{m}_{1}+\mathrm{m}_{2}\right)$ and $\omega$ is the orbital angular frequency.

Under these conditions, and using (14) and (16), one sees that the GW would be given by

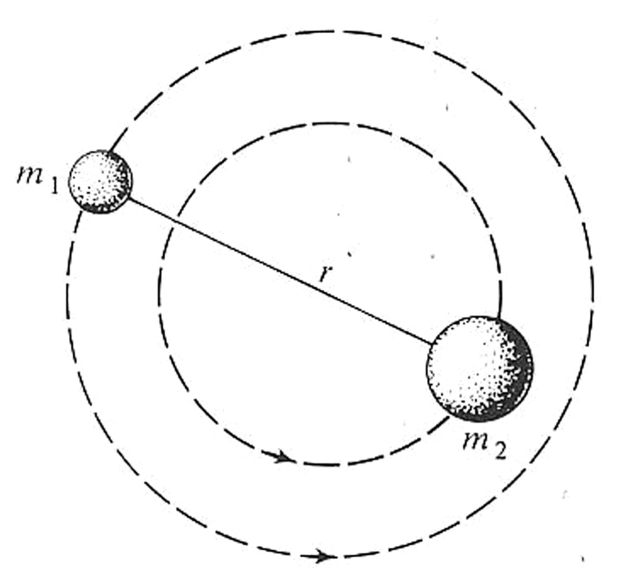

Figura 10: Binary-star system with masses $m_{1}$ and $m_{2}$ in the plane $(x, y)$.

$$
\begin{aligned}
& h_{\alpha \beta}(t, \mathbf{x}) \sim\left(2 G / c^{2} R\right)\left(\partial^{2} Q_{\alpha \beta} / \partial t^{2}\right) \\
& \sim\left(6 \mu G r^{2} / c^{2}\right) \cos (2 \omega t) / R
\end{aligned}
$$

showing that the GW frequency is $\omega_{g}=2 \omega$. Note that the GW is a tensor function, and not a scalar function as an electromagnetic wave. From (17) we verify that the strain $\mathrm{h}(\mathrm{t})$ could be written as $\mathrm{h}(\mathrm{t})$ $\sim \mathrm{h} \cos (2 \omega \mathrm{t})=\mathrm{h} \cos \left(\omega_{g} \mathrm{t}\right)$, where the GW angular frequency is $\omega_{g}=2 \omega$.

\subsection{Strain estimation}

To obtain the effective strain $\mathrm{h}$ of the $\mathrm{GW}$ that arrive at the detector it is necessary to take into account the contributions of all $h_{\alpha \beta}$ tensor components (14) to the gravitational luminosity $=\mathbf{L}_{G W}=\mathrm{dE} / \mathrm{dt}=$ 
total gravitational energy/s. The luminosity $\mathbf{L}_{G W}$ is the total energy lost by the source irradiated in all directions arriving on a spherical surface with radius $\mathrm{R}$. Thus, according to reference ${ }^{4,8}$ the energy flux $\Phi_{n}=\left(\mathrm{d}^{2} \mathrm{E} / \mathrm{dt}^{2} \mathrm{dA}\right)\left(\right.$ energy $\left./ \mathrm{m}^{2} \mathrm{~s}\right)$ that passes by the area $\mathrm{d} \mathbf{A}=\mathrm{dA} \mathbf{n}=\mathrm{R}^{2} \mathrm{dA} \mathbf{n}=\mathrm{d} \Omega \mathbf{n}$ is given by

$$
\begin{aligned}
& \Phi_{n}=\left(G / 36 \pi c^{5}\right)\left\{(1 / 2) Q_{\alpha \beta}^{2}\right. \\
& \left.-Q_{\alpha \beta} Q_{\alpha \gamma} n_{\beta} n_{\gamma}+(1 / 4)\left(Q_{\alpha \beta} n_{\alpha} n_{\beta}\right)^{2}\right\},
\end{aligned}
$$

where $\dddot{Q}_{\alpha \beta}(t)=\frac{d^{3} Q_{\alpha \beta}}{d t^{3}}$. Using (18) we can calculate the Luminosity $\mathrm{L}_{G W}$ :

$$
\begin{aligned}
L_{G W} & =<d E / d t>=R^{2} \int<\Phi_{n}>d \Omega \\
& =\left(G / 45 c^{5}\right)<Q_{\alpha \beta}^{2}>,
\end{aligned}
$$

where the brackets $<\mathrm{f}(\mathrm{t})\rangle$ is the time average of $\mathrm{f}(\mathrm{t})$.

As the energy of the GW in the radiation zone is transported by a plane wave with amplitude $h$ and orbital frequency $\omega$ the average flux value $<\Phi_{n}$ $>$ is given by: ${ }^{4}$

$$
L_{G W}=<d E / d t>\approx\left[\left(c^{3} / 8 \pi G\right) h^{2} \omega^{2}\right] 4 \pi R^{2} .
$$

Thus, with (19) and (20) let us define the following protocol to obtain h: first calculate $\mathrm{L}_{G W}$ with (19) and second calculate $\mathrm{h}$ from $\mathrm{L}_{G W}$ given by (20), from which we obtain

$$
h^{2}=\left(8 \pi G / \omega^{2} c^{3}\right)\left(L_{G W} / 4 \pi R^{2}\right) .
$$

For a binary-star we can show [6b] that

$$
\begin{aligned}
& L_{G W}=\left(G / 45^{5}\right)<Q_{\alpha \beta}^{2}>=\left(G / 45 c^{5}\right)\left[<Q_{x x}^{2}>\right. \\
& \left.+<Q_{y y}^{2}>\right]=\left(32 \mu^{2} G / 9 c^{5}\right) r^{4} \omega^{6} .
\end{aligned}
$$

From (21) and (22) we have $\mathrm{h}^{2}=\left(2 \mathrm{G} / \omega^{2} \mathrm{c}^{3}\right)\left(\mathrm{L}_{G W}\right.$ $\left./ \mathrm{R}^{2}\right)$,so that

$$
\begin{aligned}
h & =\left(8 G \mu / c^{4} \sqrt{ } 36\right) r^{2} \omega^{2} / R \\
& =\left(4 G M / R c^{4} \sqrt{ } 36\right) r^{2} \omega^{2} .
\end{aligned}
$$

As for a binary ${ }^{4} \omega^{2} \mathrm{r}^{3}=2 \mathrm{GM}, \mathrm{M}=\mathrm{m}_{1}+\mathrm{m}_{2}$, that is, $\mathrm{r}=\left(2 \mathrm{GM} / \omega^{2}\right)^{1 / 3}$; substituting this $\mathrm{r}$ value in (23) we get $h$ as a function of the orbital angular frequency $\omega(\mathrm{rad} / \mathrm{s})$ :

$$
\begin{aligned}
h & =\left(4 G M / R c^{4} \sqrt{ } 36\right)\left(2 G M / \omega^{2}\right)^{2 / 3} \omega^{2} \\
& =\left(4^{2 / 3} / \sqrt{ } 36\right)\left[(G M)^{5 / 3} / R c^{4}\right] \omega^{2 / 3} .
\end{aligned}
$$

It is important to note that the relation $\omega^{2} \mathrm{r}^{3}=$ $2 \mathrm{GM}$ can be used only when relativistic effects are very small, that is, when $\mathrm{v}_{1} / \mathrm{c}<<1$ and $\mathrm{v}_{2} / \mathrm{c}<<$ 1.

\subsection{Strain $h(\omega)$ for the event GW150914}

To estimate the strain $\mathrm{h}(\omega)$ using (24) let us take into account that [16]: distance $\mathrm{R}=410 \mathrm{Mpc}=1.3$ $\times 10^{9}$ light years $\sim 1.210^{25} \mathrm{~m}$ and that $\mathbf{B H}$ mass $\mathrm{M} \sim 30 \mathrm{M}_{\Theta}=30 \times 2 \times 10^{30} \mathrm{~kg}=610^{31} \mathrm{~kg}$. With these parameters (24) becomes

$$
h(\omega) \sim 1.110^{-23} \omega^{2 / 3},
$$

According to Fig. 8 for initial orbital frequencies ( $\mathrm{t}$ in the range $0.25<t<035)$, that is, when $\omega \sim 90$ $\pi \mathrm{rad} / \mathrm{s}$ using (25) we obtain $\mathrm{h} \sim 0.43 \times 10^{-21}$. For $\mathrm{t} \sim 0.43 \mathrm{~s}$, close to merging point, the final orbital frequencies $\mathrm{f} \sim 75 \mathrm{~Hz}$ or $\omega \sim 170 \pi \mathrm{rad} / \mathrm{s}$ we obtain $\mathrm{h} \sim 0.710^{-21}$. These estimated values of $\mathrm{h}$ are in good agreement with the observed strain $\mathrm{h}(\mathrm{t})$ shown in Figs.5-7. Since for binary system $\omega^{2} \mathrm{r}^{3}=2 \mathrm{GM}^{4,16}$ we have $\omega=\left(2 \mathrm{GM} / \mathrm{r}^{3}\right)^{1 / 2}$ showing that the orbital frequency $\omega$ increases as $r$ decreases in the merging process. This effect is verified observing Figs.5-7: up to $\mathrm{t} \approx 0.35 \mathrm{~s}$ the frequency $\omega$ is almost constant $\omega \approx 90 \pi \mathrm{rad} / \mathrm{s}$ and or $0.43>t>0.35 \omega$ increases becoming $\omega \approx 170 \pi \mathrm{rad} / \mathrm{s}$, before the merging point, at $\mathrm{t}=0.43 \mathrm{~s}$.

\subsection{Strain $h(\omega)$ for the Event GW151226}

In this event we have [20] $\mathrm{R} \approx 440 \mathrm{Mpc}$ and the $\mathbf{B H}$ masses, $\mathrm{m}_{1} \approx 14.2 \mathrm{M}_{\Theta}$ and $\mathrm{m}_{1} \approx 7.5 \mathrm{M}_{\Theta}$. Thus, with these parameters (24) becomes

$$
h(\omega) \sim 0.7310^{-23} \omega^{2 / 3} .
$$

During signal detection the GW frequencies $\mathrm{f}_{g}$ increased from 35 to $450 \mathrm{~Hz}$. According to Fig.9 in the "inspiral"stage we see that for 3 different times, $\mathrm{h} \sim 0.1710^{-21}, \mathrm{~h} \sim 0.18 \times 10^{-21}$ and close to the merging time $\mathrm{t} \sim 0 \mathrm{~s}, \mathrm{~h} \sim 0.34 \times 10^{-21}$. As the $\mathrm{GW}$ frequencies $\mathrm{f}_{g}$ are two times larger than the orbital frequencies, for initial orbital frequencies 
$\omega \approx 17.5 \pi \mathrm{rad} / \mathrm{s}$ we verify using $(26)$ that $\mathrm{h} \sim$ $0.14 \times 10^{-21}$ in good agreement with Fig.9. Close to the merging point $\mathrm{t} \sim 0 \mathrm{~s}$ when $\mathrm{f}_{g} \approx 300 \mathrm{~Hz}$, that is, when $\omega \approx 150 \pi \mathrm{rad} / \mathrm{s}(26)$ gives $\mathrm{h} \sim 0.60 \mathrm{x}$ $10^{-21}$. So, at the merging point our estimates are two times larger than the measured strain. Probably this occurs because the $\mathrm{BH}$ velocities are large (see second row of Fig.9), that is, v/c $\sim 0.4 \sim 0.5$ and our non-relativistic approach $\omega^{2} \mathrm{r}^{3}=2 \mathrm{GM}^{4,16,20}$ is not valid.

\subsection{Strain $h$ as a function of time}

Let us give a rough description of the strain as a function of the time $\mathrm{h}=\mathrm{h}(\mathrm{t})$ as it is seen in Figs. 7 and 8 in the "inspiral"stage. According to (17) in the initial or "inspiral"phase we have $\mathrm{h}(\mathrm{t}) \sim \mathrm{h}$ $\cos \left(\omega_{g} \mathrm{t}\right)$, where $\omega_{g}=2 \pi \mathrm{f}_{g}$ is the angular frequency $(\mathrm{rad} / \mathrm{s})$ of the GW. As our estimated values $\mathrm{h}(\omega)$ for GW150226 and GW 151226 are given by $\mathrm{h}(\omega)$ $=\mathrm{a} 10^{-23} \omega^{2 / 3}$, with $\mathrm{a}=1.1 \times 10^{-23}$ and $\mathrm{a}=0.73 \mathrm{x}$ $10^{-23}$, respectively, we write

$$
\begin{aligned}
h(t) & =a \omega(t)^{2 / 3} \cos \left[\omega_{g}(t) t\right] \\
& =a \omega(t)^{2 / 3} \cos [2 \omega(t) t],
\end{aligned}
$$

remembering that $\omega_{g}=2 \omega$. Now, taking $\omega(\mathrm{t})^{2} \mathrm{r}(\mathrm{t})^{3}$ $=2 \mathrm{GM}=$ constant $=\omega_{o}^{2} \mathrm{r}_{o}^{3}$, where $\omega_{o}$ and $\mathrm{r}_{o}$ are the "initial values"(very far from the merging point) we see that $\omega(\mathrm{t})=\omega_{o}\left[\mathrm{r}_{o} / \mathrm{r}(\mathrm{t})\right]^{3 / 2}$. Due to the GW emission $[3,9,11,12]$, the mechanical energy of the binary decreases with time and the distance $\mathrm{r}(\mathrm{t})$ decreases according to the law $\mathrm{r}(\mathrm{t})=\mathrm{r}_{o}(1-\mathrm{t} / \tau)^{1 / 4}$, where $\tau$ is the "spiral time". For neutron stars and $\mathrm{BH}$ binaries, according to Box 36.3 of reference [3], $\tau$ changes from $\sim 3.2 \mathrm{yr}$ up to $0.10 \mathrm{msec}$, when $\mathrm{r}_{o}$ goes from $10^{4} \mathrm{~km}$ up to $10 \mathrm{~km}$. So, as the distance $\mathrm{r}(\mathrm{t})$ decreases the rotational frequencies $\omega(\mathrm{t})$ increases as $\omega(\mathrm{t})=\omega_{o} /[1-\mathrm{t} / \tau]^{3 / 8}$ as $\mathrm{t}$ becomes close to $\tau$. When the $\mathrm{BH}$ begins to merge, that is, when $\mathrm{t} \rightarrow \tau$ , $\omega(\mathrm{t})$ can reach extremely high values, and the GW amplitude $\mathrm{h}(\mathrm{t})$, according to (27), reaches its peak ${ }^{20}$ (see the SNR third row of Fig.8).

\section{Acknowledgements}

The authors thank a critical reading of the paper and illustrative suggestions by Prof. Dr. J. A. de Freitas Pacheco, member of the LIGO-VIRGO collaboration. The authors also thank the librarian
Virginia de Paiva for her assistance in the pursuit of various texts used as references in this article.

\section{Referências}

[1] K.S. Thorne, Rev. Mod. Phys. 52, 285 (1980).

[2] K.S. Thorne, Astrophys. J. 158, 997 (1969).

[3] C.W. Misner, K.S. Thorne and J.A. Wheeler, Gravitation (Freeman, San Francisco, 1970).

[4] L.D. Landau et E.M. Lifchitz, Théorie du Champ (Mir, Moscow, 1964).

[5] M.Cattani, RBEF 20, 27 (1998).

[6] M.Cattani, arXiv:1005.4314 (2010).

[7] M.Cattani, arXiv:1007.0140 (2010).

[8] M.Cattani, http://arxiv.org/abs/1001.2518 (2009).

[9] M.Cattani, http://arxiv.org/abs/1003.2105 (2010).

[10] M.Cattani, http://arxiv.org/abs/1004.2470 (2010).

[11] I.R. Kenyon, General Relativity (Oxford University Press, Oxford, 1990).

[12] H.C. Ohanian, Gravitation and Spacetime (W.W. Norton, New York, 1976).

[13] E. Hecht, Optics (Addison \& Wesley Longman, Reading, 1998).

[14] The Virgo Project, Nuclear Instr. and Meth. in Phys. Res. A289, 518 (1990).

[15] A. Giazotto, S. Bonnazzola, and E. Gourgulhon, arXiv: astro-ph/9611188v1 (1996); E. Gourgulhon and S. Bonnazzola, arXiv: astro-ph/9605150v1 (1996).

[16] B.P. Abbott, LIGO Scientific Collaboration and Virgo Collaboration, Phys. Rev. Lett. 116, 061102 (2016).

[17] https://en.wikipedia.org/wiki/First_ observation_of_gravitational_waves

[18] https://en.wikipedia.org/wiki/Virgo_ interferometer.

[19] J.A. de Freitas Pacheco, Research in Astron. Astrophys. 10, 1071 (2010). See also https://en. wikipedia.org/wiki/Binary_black_hole

[20] B.P. Abbott, LIGO Scientific Collaboration and Virgo Collaboration, Phys. Rev. Lett. 116, 241103 (2016). 